\title{
VARIĀNCIA DOS CONTRASTES CLÁSSICOS NOS EXPERIMENTOS COM PARCELAS SUBDIVIDIDAS EM BLOCOS INCOMPLETOS BALANCEADOS
}

\section{Antonio Francisco Iemma}

Departamento de Bioestatística. Instituto Bāsico de Biologia Médica a Agrícola. UNESP. Botucatu, SP.

Humberto de Campos

Departamento de Matemātica e Estatīstica. Escola Superior de Agricul tura "Luiz de Queiroz". USP. Piracicaba, SP.

RESUMO

Neste estudo analisou-se o comportamento dos contrastes clássicos nos experimentos em parcelas subdivididas com tratamentos principais dispostos em blocos incompletos balanceados. Considerou-se a existēncia de correlação constante $\rho$ entre subparcelas de uma mes ma parcela e independéncia entre subparcelas de parcelas distintas. A variāncia dos contrastes básicos, para esse tipo de experimentos, portou-se ora como nos ensaios em BIB, ora como nos ensaios em par celas subdivididas delineados em blocos (completos) casualizados.

\section{SUMMARY}

IEMMA, A.F., and CAMPOS, H., 1981. Variance of the classical contrasts at the split-plot experiments in balanced incomplete block designs. Ciência e Natura (3):21-27.

In this paper the conduct of the split-plot design with the main treatments disposed in balanced incomplete block, was analised. Moreover, the presence of constant correlaction, $\rho$, between two sub plots of same plot, and independence among sub plots of the distincts plots, was considered. The variances for the basics contrasts of this experimencs sometimes were analogous at the variances in the incomplete block designs and other time analogous at the variances in the splitplot experiments in the randomized block design.

INTRODUÇAOO

Os experimentos com parcelas subdivididas em blocos (com pletos) casualizados tēm sido de grande valia na pesquisa agropecuá ria, por sua alta eficiēncia e grande simplicidade. No entanto,exis tem situações nas quais a formação dos blocos completos torna-se pra ticamente impossive1. Por exemp10, PIMENTEL GOMES (8), cita um expe rimento com suinos no qual se deseja testar um grande número de tra tamentos. Considerando-se que para experimentos desse tipo está con sagrado o uso de leitegada como bloco, o experimentador tem diante 
de si um impasse: ou diminue o número de tratamentos ou não toma lei tegada como bloco. Na primeira escolha perderá a oportunidade de com parar os tratamentos eliminados com os demais, na segunda fatalmente perderá em eficiência, dada a menor homogeneidade inerente aos "no vos" blocos.

Assim como esta, existem inūmeras outras situações na pes quisa agropecuária, que sugerem o uso dos ensaios com parcelas subdi vididas delineados em blocos incompletos.

Considerou-se, neste estudo, que para o uso adequado de tais experimentos seria desejāvel conhecer-se o comportamento dos contrastes básicos envolvendo tratamentos principais, tratamentos se cundārios e ambos. Assim, dada a extensão do tema, tomou-se o caso em que os tratamentos principais estivessem dispostos em blocos in completos balanceados.

\section{DESENVOLVIMENTO TEORICO}

Tomou-se o modelo linear

$$
y_{i j s}=m+t_{i}+b_{j}+e_{k(i j)}+t^{\prime} s+\left(t t^{\prime}\right)_{i s}+e_{i j s}
$$

onde, para $i=1,2, \ldots, v ; j=1,2, \ldots, a ; s=1,2, \ldots, u$

$y_{i j s}$ é o valor observado na subparcela que recebeu o s-ēsi mo tratamento secundārio dentro do i-ēsimo tratamento principal, no j-ēsimo bloco; m ē a média geral; $t_{j} \bar{e}$ o efeito do i-ésimo tratamen to principal; b $b_{j} \bar{e}$ o efeito do j-ēsimo bloco; e $k$ (ij) é o erro atri buido à k-ēsima parcela do bloco $j$, que recebeu o i-ésimo tratamen to principal; t's é o efeito do s-ésimo tratamento secundārio;(tt') ${ }_{j s}$ é o efeito da interação entre o i-ésimo tratamento principal e o s-és mo tratamento secundārio; e ${ }_{i j s}$ é o erro atribuido à observação $y_{i j s}$.

Ademais. considerou-se como em CHAKRABARTI (1), COCHRAN e COX (3), DINIZ (4), IEMMA (5) e LEAL (7), dentre outros, a existēn cia de uma correlação constante entre subparcelas de uma mesma par cela e independēncia entre subparcelas de parcelas distintas, resul tando:

$$
\operatorname{Cov}\left(y_{i j s^{\prime}} ; y_{i} j^{\prime} s^{\prime}\right)=\left\{\begin{array}{l}
\sigma^{2} ; \text { se } i=i^{\prime}, j=j^{\prime}, s=s^{\prime} \\
\rho \sigma^{2} ; \text { se } i=i^{\prime}, j=j^{\prime}, s \neq s^{\prime} \\
0 \text {; em outros casos }
\end{array}\right.
$$

Naturalmente a opção pelo modelo proposto, em lugar dos mo delos menos restritivos das anālises de perfill, está condicionada aos testes de homogeneidade e de uniformidade das matrizes covariāncias, conforme descrito em COCHRAN (2), IEMMA (5) e LEAL (7), dentre ou tros. 
No modelo linear geral $Y=X \theta+\varepsilon$, partiu-se a matriz $X$ em $x=\left[\begin{array}{l:l:l:l:l}x_{1} & x_{2}: x_{3} & x_{4} & x_{5}\end{array}\right]$, onde $x_{1}, \ldots, x_{5}$ têm (urv) linhas e (1), (v), (a), (u) e (uv) colunas respectivamente e estão associa das aos coeficientes relativos à média geral, tratamentos principais, blocos, tratamentos secundārios e interação.

Do desenvolvimento do sistema de equações normais $X^{\prime} X \tilde{\theta}=X^{\prime} Y$, obteve-se

$$
\left[\begin{array}{lllll}
n & X & y & z & w \\
x^{\prime} & R & N & P & S \\
y^{\prime} & N^{\prime} & A & K & V \\
z^{\prime} & P^{\prime} & K^{\prime} & U & H \\
w^{\prime} & S^{\prime} & V^{\prime} & H^{\prime} & L
\end{array}\right]\left[\begin{array}{c}
\hat{m} \\
\bar{\tau} \\
\hat{B} \\
\hat{\tau} * \\
\hat{\delta}
\end{array}\right]=\left[\begin{array}{c}
X^{\prime}{ }_{1} Y \\
X^{\prime}{ }_{2} Y \\
X^{\prime}{ }_{3} Y \\
X^{\prime}{ }_{4} Y \\
X^{\prime}{ }_{5} Y
\end{array}\right]=\left[\begin{array}{c}
G \\
T \\
B \\
T^{*} \\
\Delta
\end{array}\right]
$$

onde $\hat{m}, \hat{\tau}, \hat{\beta}, \tilde{\tau}^{*}$ e $\widehat{\delta}$ são vetores dos efeitos estimados da média geral, tratamentos principais ajustados para efeitos de blocos, tratamen tos secundários e pares $\left(t t^{\prime}\right)_{\text {is }}$, respectivamente.

$\mathrm{Na}$ determinação das matrizes de dispersão para os parâme tros bāsicos, tomou-se a definição:

$$
D(\hat{p})=E\left\{[\hat{p}-E(\hat{p})|| \hat{p}-E(\hat{p})]^{\prime}\right\}
$$

Assim, para tratamentos principais, obteve-se:

$D(\tilde{\tau})=E\left\{M^{-1}\left(X_{2}^{\prime}-N A^{-1} X_{3}{ }_{3}\right) \varepsilon \varepsilon^{\prime}\left(X_{2}-X_{3} A^{-1} N^{\prime}\right) M^{-1}\right\}$

Mas, se são verificadas as pressuposições do mode10

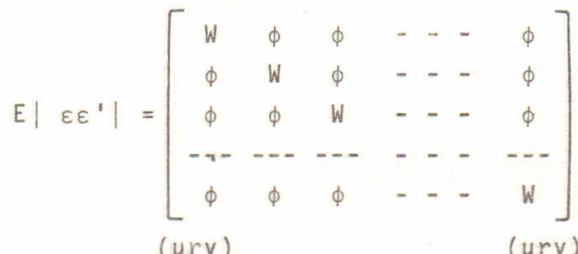

onde

$$
\begin{aligned}
& W=\left[\begin{array}{cccc}
\sigma^{2} & \rho \sigma^{2} & \cdots & \rho \sigma^{2} \\
\rho \sigma^{2} & \sigma^{2} & \cdots & \rho \sigma^{2} \\
\cdots-\cdots & \cdots & \cdots \\
\rho \sigma^{2} & \rho \sigma^{2} & \cdots & \sigma^{2}
\end{array}\right] \\
& \text { (u) } \\
& \text { (u) } \\
& \begin{aligned}
\therefore D(\bar{\tau})= & \frac{K^{2}}{\lambda^{2} u^{2} v^{2}}[1+(u-1) \rho] \sigma^{2}\left(X_{2}{ }^{\prime}-N A^{-1} X^{\prime}{ }_{3}\right)\left(X_{2}-X_{3} A^{-1} N^{\prime}\right) \\
& \frac{K^{2}}{\lambda^{2} u^{2} v^{2}}[1+(u-1) \rho] \sigma^{2}\left(R-N A^{-1} N^{3}\right)
\end{aligned}
\end{aligned}
$$


e, fazendo-se como de modo usual na teoria dos blocos incompletos $C=R-N A^{-1} N^{\prime}$, obteve-se

$$
D(\bar{\tau})=M^{-1} C M^{-1}[1+(u-1) \rho] \sigma^{2}
$$

Donde

$$
d_{i j}{ }^{\prime}=\left\{\begin{array}{l}
\frac{k(v-1)}{\lambda u v^{2}}[1+(u-1) \rho] \sigma^{2} \text {, se } i=i \\
-\frac{k}{\lambda u v^{2}}[1+(u-1) \rho] \sigma^{2} \text {, se } i \neq i
\end{array}\right.
$$

Assim, para variância da diferença entre duas médias de $\underline{e}$ feitos estimados de tratamentos principais, observou-se

$$
v\left(\hat{t}_{i}-\hat{t}_{i}{ }^{\prime}\right)=\frac{2 k}{\lambda u v}[1+(u-1) \rho] \sigma^{2}
$$

que é um resultado coerente com a teoria dos ensaios em blocos incom pletos balanceados. Notou-se, por exemplo, que se $u=1$, então não hā o "split plot" e o resultado ē exatamente aquele fornecido por P $\underline{I}$ MENTEL GOMES (8) para $V\left(\bar{t}_{j}-\bar{t}_{j}\right.$ ) quando o ensaio é efetuado em BIB.

Tomando-se os resultados obtidos por IEMMA (6) obteve-se:

$\bar{v}\left(\hat{t}_{i}-\hat{t}_{j^{\prime}}\right)=\frac{2 K}{\lambda u V} Q M \operatorname{Res}(a)$

De modo anālogo, para tratamentos secundārios obteve-se:

$$
\begin{aligned}
D\left(\hat{\tau}^{*}\right) & =E\left\{\left[\hat{\tau}^{*}-E\left(\hat{\tau}^{*}\right)\right]\left[\hat{\tau}^{*}-E\left(\hat{\tau}^{*}\right)\right]^{\prime}\right\} \\
& =\frac{1}{r^{2} v^{2}} x^{\prime}{ }_{4} E\left[\varepsilon \varepsilon^{\prime}\right] x_{4} \\
\therefore D\left(\bar{\tau}^{*}\right) & =\frac{1}{r v}(u)^{W}(u)
\end{aligned}
$$

donde

$$
\begin{gathered}
d{ }^{*}{ }_{i j}{ }^{\prime}=\left\{\begin{array}{l}
\frac{\sigma^{2}}{r v}, \text { se } i=i^{\prime} \\
\frac{\rho \sigma^{2}}{r v}, \text { se } i \neq i^{\prime}
\end{array}\right. \\
\text { Então } V\left(\bar{t}^{\prime}{ }_{s}-\bar{t}^{\prime}{ }_{s^{\prime}}\right)=\frac{2}{r v}(1-\rho) \sigma^{2}
\end{gathered}
$$

e, usando-se os resultados obtidos por IEMMA (5), obteve-se:

$$
\hat{v}\left(\bar{t}^{\prime}{ }_{s}-\bar{t}^{\prime} s^{\prime}\right)=\frac{2}{r V} Q M \operatorname{Res}(b)
$$

que é um resultado idêntico aquêles encontrados na literatura para esse tipo de contraste, quando o ensaio é realizado em parcelas sub divididas, com tratamentos principais dispostos em blocos (comple tos, casualizados. 
No tocante à interação, tomou-se

$D(\hat{\delta})=E\left\{[\hat{\delta}-E(\hat{\delta})][\hat{\delta}-E(\hat{\delta})]^{\prime}\right\}$

cujo desenvolvimento resultou

$$
D(\delta)=\frac{1}{r^{2}} \Gamma E\left(\varepsilon \varepsilon^{\prime}\right) \Gamma^{\prime}
$$

onde a matriz $\Gamma$ tem dimensões (uv) X. (uv) e é tal que

$$
\gamma_{i j}=\left\{\begin{array}{l}
\Gamma_{1}, \text { se o tratamento principal } i \text { ocorre no bloco } j \\
\Gamma_{2}, \text { caso conträrio }
\end{array}\right.
$$

com

$$
\Gamma_{1}=\frac{1}{u v}\left[\begin{array}{ccccc}
(u-1)(v-1) & -(v-1) & -\cdots & - & (v-1) \\
-(u-1) & (u-1)(v-1) & -- & - & (v-1) \\
-- & --- & -- & -- \\
-(v-1) & -(v-1) & -- & (u-1)(v-1)
\end{array}\right]
$$

e

$$
\Gamma_{2}=\frac{1}{u v}\left[\begin{array}{cccc}
-(u-1) & 1 & -- & 1 \\
1 & -(u-1) & -- & 1 \\
-- & -- & -- & --(u-1) \\
1 & 1 & -- & -(u)
\end{array}\right]
$$

(u)

(u)

obtendo-se, desse modo

$$
D(\bar{\delta})=\frac{(1-\rho) \sigma^{2}}{u r v}\left[\begin{array}{ccccc}
\Gamma_{1} & \Gamma_{2} & \Gamma_{2} & \cdots & \Gamma_{2} \\
\Gamma_{2} & \Gamma_{1} & \Gamma_{2} & -- & \Gamma_{2} \\
----- & -- & -- & -- \\
\Gamma_{2} & \Gamma_{2} & \Gamma_{2} & -- & \Gamma_{1}
\end{array}\right]
$$

(u)

(uv)

Assim, a variância para a diferença entre duas mēdias de. efeitos estimados de tratamentos secundários dentro de um mesmo tra tamento principal, ficou

$$
\begin{aligned}
& V\left(\widehat{\delta}_{i s}-\widehat{\delta}_{i s^{\prime}}\right)=2[(u-1)(v-1)+(v-1)] \frac{(1-\rho)}{u r v} \sigma^{2} \\
& \therefore V\left(\widehat{\delta}_{i s}-\widehat{\delta}_{i s^{\prime}}\right)=\frac{2}{r}\left(1-\frac{1}{v}\right)(1-\rho) \sigma^{2}
\end{aligned}
$$

que é um resultado idêntico aquêle apresentado por CHAKRABARTI (1) para esse tipo de contraste, quando o experimento em parcelas subdi vididas ē delineado em blocos (completos) casualizados. 
E, de modo análogo aos anteriores

$\hat{V}\left(\hat{\delta}_{i s}-\hat{\delta}_{i s^{\prime}}\right)=\frac{2}{r}\left(1-\frac{1}{v}\right)$ QM Res (b)

Para a variāncia da diferença entre duas médias de efeitos estimados de tratamentos principais dentro de um mesmo tratamento se cundārio, foi obtida:

$$
\begin{aligned}
& V\left(\hat{\delta}_{i s}-\widehat{\delta}_{i ' s}\right)=\frac{2}{r}\left(1-\frac{1}{u}\right)(1-p) \sigma^{2} \\
& \therefore \hat{V}\left(\widehat{\delta}_{i s}-\delta_{i} s_{s}\right)=\frac{2}{r}\left(1-\frac{1}{u}\right) Q M \operatorname{Res}(b)
\end{aligned}
$$

Neste resultado houve concordāncia com CHAKRABARTI (1), pa ra ensaios em parcelas subdivididas delineados em blocos (completos) casualizados, no entanto houve discordāncia com PIMENTEL GOMES (8) que apresenta como solução uma ponderação entre resíduos (a) e (b). CONCLUSOES

Do estudo das variâncias das funções lineares estimāveis, atravēs das matrizes de dispersão determinadas neste estudo para os parãmetros bāsicos, concluiu-se que:

1. A variāncia obtida para contrastes entre mēdias de tra tamentos principais, mostrou-se anāloga āquelas obtidas para contras tes entre médias de tratamentos, nos ensaios em BIB.

2. As variâncias obtidas para contrastes entre médias de tratamentos secundārios, entre médias de tratamentos secundārios "den tro" de um mesmo tratamento principal e entre médias de tratamentos principais dentro de um mesmo tratamento secundārio, foram coerentes com aquelas encontradas na bibliografia dos ensaios em parcelas sub divididas com tratamentos principais dispostos em blocos (completos) balanceados, para autores que consideram correlação constante entre sub parcelas de uma mesma parcela.

BIBLIOGRAF I A

1. CHAKRABARTI, M.C. Mathematies of Design an Analysis of Experị ments. 1. ed. Asia Publishing House, London, 1962, 120 p.

2. COCHRAN, W.G. Testing a linear relation among variances. Biome tries, Raleigh, 7 : 17-32, 1951.

3. COCHRAN, W.G. e COX, G.M. Disenos Experimentales. 3. ed., Trillas, México, 1976, $661 \mathrm{p}$.

4. DINIZ, U.D. Análise de Experimentos com Parcelas Medidas Sucessi vamente no Tempo. ESALQ/USP, Piracicaba, 1980, 104 p. (Tese de Doutoramento).

5. IEMMA, A.F. Anälise de experimentos em parcelas subdivididas com tratamentos principais dispostos em blocos incompletos balan 
ceados. ESALQ/USP, Piracicaba, 1981, 145 p. (Tese de Doutora mento).

6. IEMMA; A.F. Comparações Mültiplas pelo Critério de Tukey nos en saios em parcelas subdivididas com delineamentos incompletos. I - BIB. ANAIS DA X JORNADA CIENTIFICA DO CAMPUS DE BOTUCATU, 1981.

7. LEAL, M.L.S. Análise de dados experimentais com medidas repetidas. U.F.B., Brasîlia, 1979, 99 p. (Dissertação de Mestrado).

8. PIMENTEL GOMES, F. Curso de Estatistica Experimental. 6a. ed., No be 1, Piracicaba, 1976, 430 p.

9. RAO, C.R. Advanced Statistical Methods in Biometric Research. 1. ed., Macmillan Publishing Co, New York, 1952, 390 p.

Recebido em novembro, 1981; aceito em novembro, 1981. 
